\title{
Urea cycle disorder presenting as bilateral mesial temporal sclerosis - an unusual cause of seizures: a case report and review of the literature
}

\author{
Furene Sijia Wang ${ }^{1,2^{*}}$ (D) Denise Li Meng Goh ${ }^{1,2}$ and Hian Tat Ong ${ }^{1,2}$
}

\begin{abstract}
Background: Urea cycle disorders are secondary to defects in the system converting ammonia into urea, causing accumulation of ammonia and other byproducts which are neurotoxic. Ornithine transcarbamylase deficiency is the most common of the urea cycle disorders and frequently presents with coma or seizures during hyperammonemia. However, seizures can also occur without metabolic decompensation.

Case presentation: We describe a 23-year-old Chinese woman with urea cycle disorder who presented with confusion due to focal seizures arising from the left frontotemporal region. Interestingly, her ammonia levels remained normal during the seizures. Neuroimaging showed bilateral mesial temporal sclerosis. Her seizures were successfully controlled with two anti-epileptic medications.

Conclusions: This case adds evidence of the predisposition of the temporal lobe to injury in urea cycle disorder. Urea cycle disorder can lead to mesial temporal sclerosis which leads to increased susceptibility of patients to seizures regardless of their metabolic state.
\end{abstract}

Keywords: Urea cycle disorder, Mesial temporal sclerosis, Hyperammonemia, Magnetic resonance imaging

\section{Background}

Urea cycle disorders (UCD) are a group of inborn errors of metabolism caused by the dysfunction of any of the six enzymes or two transport proteins involved in urea biosynthesis. The six enzymes are carbamoyl phosphate synthetase I, N-acetylglutamate synthetase, ornithine transcarbamylase, argininosuccinic acid synthetase, argininosuccinate lyase, and arginase. The two amino acid transport defects are ornithine translocase and aspartate/glutamate carrier. All the UCDs are inherited as autosomal recessive disorders, except for ornithine transcarbamylase (OTC) deficiency. A defect in urea synthesis results in hyperammonemia, which disrupts the aquaporin system and increases astrocyte glutamine synthesis causing brain edema and raised intracranial pressure [1]. The severity of the UCD is influenced by the

\footnotetext{
* Correspondence: furene_wang@nuhs.edu.sg

${ }^{1}$ Khoo Teck Puat - National University Children's Medical Institute, National University Hospital, Singapore, Singapore

2Department of Paediatrics, Yong Loo Lin School of Medicine, National University of Singapore, Singapore, Singapore
}

position of the defective protein in the pathway and the severity of the defect. The clinical presentation is variable with the most severe forms presenting in the neonatal period with seizures, coma, and multiorgan failure. The seizures can be subclinical during acute hyperammonemic episodes. Milder forms of UCD can present at any age with metabolic decompensation or more subtle symptoms such as developmental delay, and behavioral or psychiatric symptoms. However, with good metabolic control of the underlying disease, resultant epilepsy is rare in the course of the disorder [2].

We describe a patient with UCD who presented with seizures despite having good control of her ammonia levels.

\section{Case presentation}

Our patient is a 23-year-old Chinese woman with UCD who presented with seizures 2 years after the latest episode of metabolic decompensation. She was the second child of a non-consanguineous union. Her elder sister and parents were well and there was no history of early

(c) The Author(s). 2018 Open Access This article is distributed under the terms of the Creative Commons Attribution 4.0 International License (http://creativecommons.org/licenses/by/4.0/), which permits unrestricted use, distribution, and 

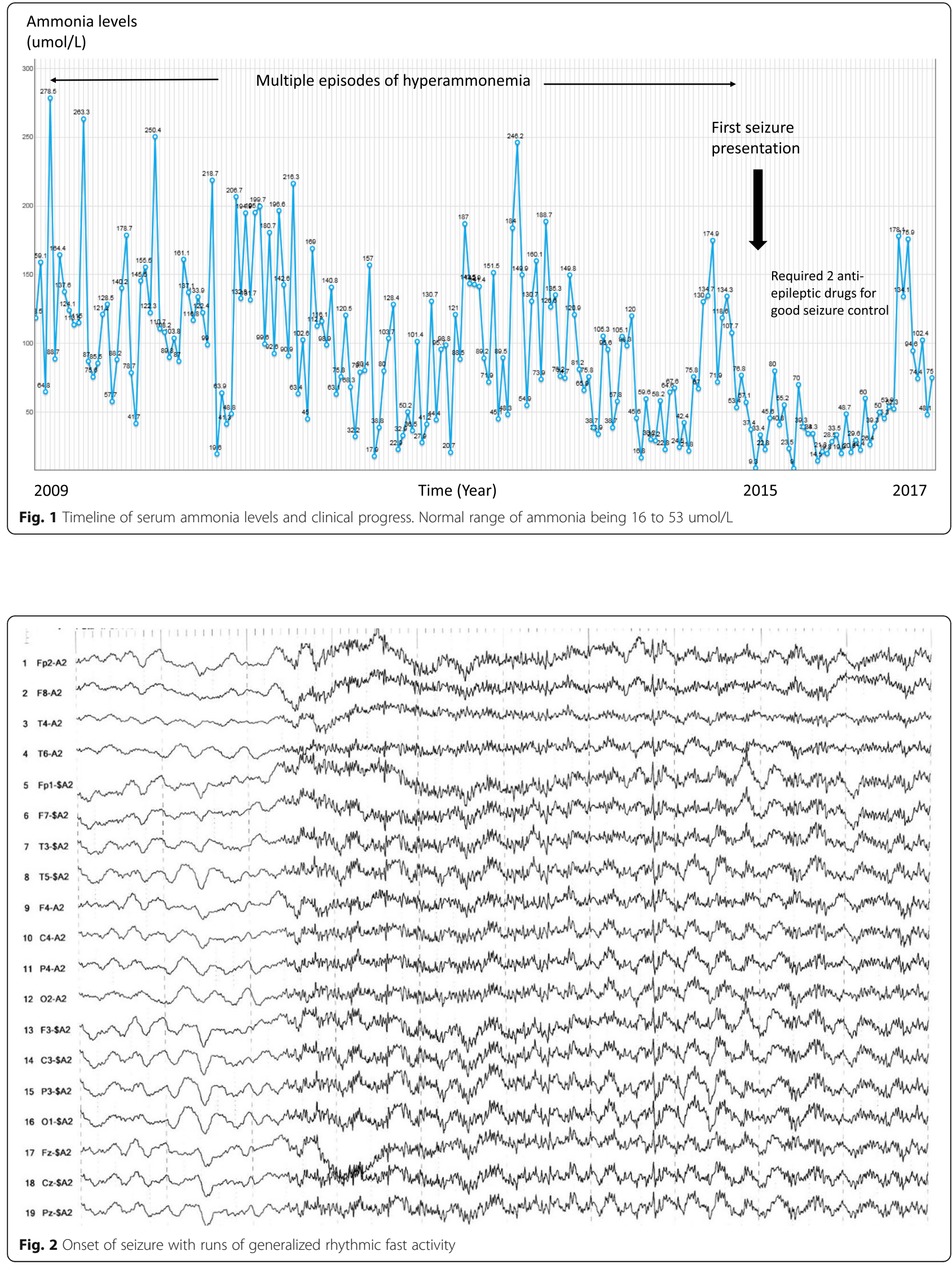
deaths in the family, especially male family members. She was delivered at full term via an emergency caesarean section for failure to progress and breech position. Her Apgar was 7 at 1 minute and 8 at 5 minutes, probably due to prolonged maternal anesthesia.

She presented at 14 months of age with gross motor delay and intermittent vomiting after meals. She was alert and interactive. However, she was ataxic and her lower limbs were hypotonic with decreased power and brisk reflexes. The tone, power, and reflexes were normal in her upper limbs. She had intention tremors of the upper limbs. Computed tomography (CT) of her brain did not show any intracranial abnormalities. Her plasma ammonia level was markedly elevated at $327 \mathrm{umol} / \mathrm{L}$ (normal range 16 to $53 \mathrm{umol} / \mathrm{L}$ ). She was treated with intravenously administered sodium benzoate with improvement in the hyperammonemia. She was diagnosed as having OTC deficiency in view of hyperammonemia, elevated glutamine at $1237 \mathrm{umol} / \mathrm{L}$ (normal range 400 to $700 \mathrm{umol} / \mathrm{L}$ ), and elevated urinary orotic acid at $110 \mathrm{mmol} / \mathrm{mol}$ creatinine (normal range 0.5 to $3.3 \mathrm{mmol} /$ mol creatinine). Her citrulline level was normal at 17 umol/L (normal range 5 to $60 \mathrm{umol} / \mathrm{L}$ ). Sequencing of the OTC gene did not detect any pathological variant. The inability to identify a pathological variant by sequencing is not unusual. Pathological point mutation variants are found in approximately $80 \%$ of patients with enzymatically confirmed OTC deficiency. The remaining patients either have variants in the regulatory regions, variants within the introns, or have large deletions, all of which would not be detected by the sequencing that was done in this patient.

Our patient had multiple hospital admissions from diagnosis to 19 years of age, due to episodes of metabolic decompensation with plasma ammonia levels ranging between 157 and 278 umol/L (Fig. 1). These episodes occasionally occurred due to suboptimal compliance to protein-restricted diet, but most of the episodes occurred without any obvious trigger. She responded each time to intravenously administered sodium benzoate or sodium phenylbutyrate, L-arginine and $10 \%$ dextrose infusion with normalization of the ammonia levels. She would then resume her protein-restricted diet, with orally administered sodium benzoate and citrulline. Functionally, she was independent in the activities of daily living. However, she was intellectually impaired with an IQ score of 40 and received special education.

She presented with the first episode of seizure at 21 years of age. She did not have previous febrile seizures in childhood. There was no family history of epilepsy. She had altered mental state and incoherent

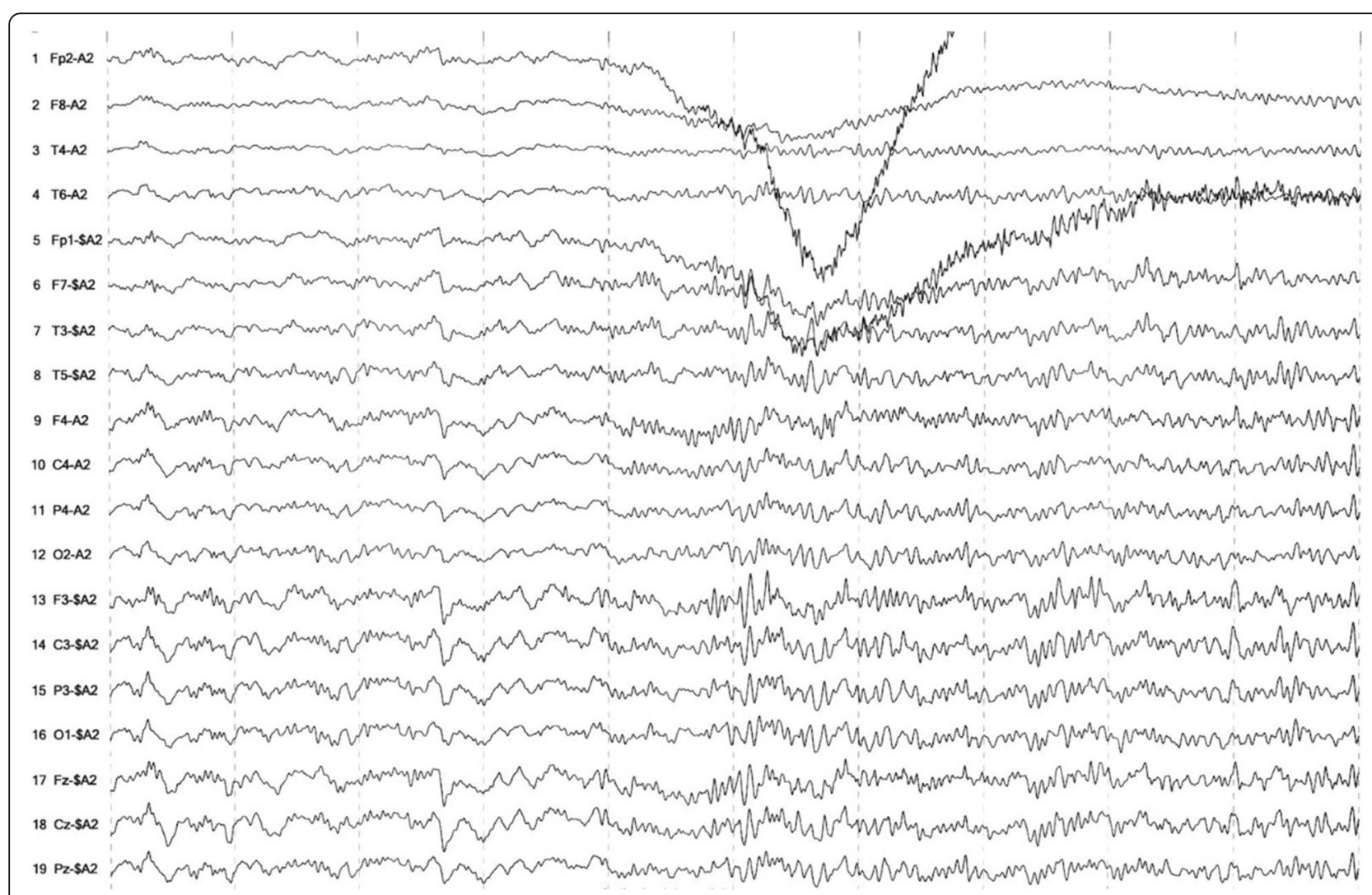

Fig. 3 Onset of seizure from left frontotemporal (F3 > T3) region with subsequent generalization 
speech on presentation. The plasma ammonia levels remained normal, ranging from 16 to $45 \mathrm{umol} / \mathrm{L}$.

An electroencephalogram (EEG) recorded non-convulsive seizures with the onset of rhythmic fast activity (Fig. 2), occasionally starting at the left frontotemporal region before becoming generalized (Fig. 3). These were associated with clinical manifestations of oral automatisms, impaired consciousness, and right-sided head turn.

Magnetic resonance imaging (MRI), which was performed 2 days after the first seizure presentation, showed T2/fluid-attenuated inversion recovery (FLAIR) signal hyperintensity in bilateral parahippocampal gyri with loss of gray-white matter differentiation and dilatation of bilateral temporal horns suggestive of hippocampal atrophy, due to mesial temporal sclerosis (Fig. 4). There was also restricted diffusion noted in the parahippocampal regions on both sides (Fig. 5). There was no prior MRI imaging performed. She was started on levetiracetam and pregabalin as these anti-epileptic drugs had minimal drug-drug interaction. Pregabalin was added on as she continued to have breakthrough seizures with levetiracetam. The use of sodium valproate is contraindicated in UCDs as it predisposes to hyperammonemia. Her seizures remained well controlled with the use of the two anti-epileptic medications.

\section{Discussion}

Our patient's neuroimaging finding of bilateral mesial temporal sclerosis was unusual as acquired mesial temporal sclerosis is commonly known to be a consequence of long-standing refractory epilepsy rather than the cause of seizures. She remained seizure free during multiple episodes of hyperammonemia. However, when the clinical seizures occurred, she was not in metabolic

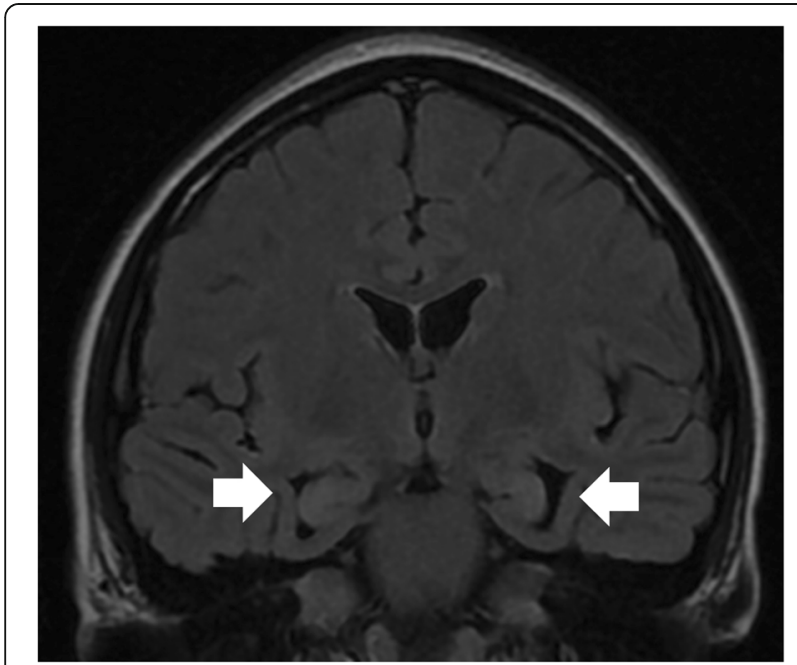

Fig. 4 Coronal T2 fluid-attenuated inversion recovery showing signal hyperintensity in bilateral parahippocampal gyri (arrowed) with loss of gray-white matter differentiation due to mesial temporal sclerosis

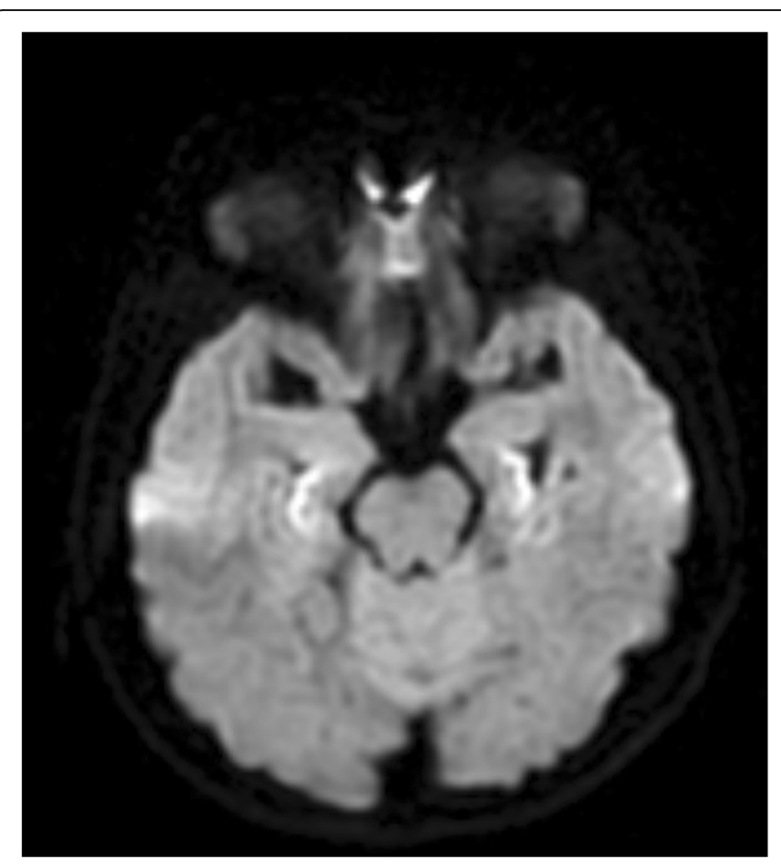

Fig. 5 Restricted diffusion in parahippocampal regions on both sides seen on diffusion weighted image sequences

decompensation. The temporal relation leads us to postulate that the neuroimaging findings were a result of metabolic abnormalities during her childhood and adolescent years or due to undiagnosed subclinical seizures during metabolic decompensation.

The predilection of the temporal lobes to injury from hyperammonemia is well-described. There is a specific pattern of brain injury in UCDs [3]. Acute hyperammonemia causes reversible changes involving the deep sulci of the insular and perirolandic regions. MRI studies have described hypomyelination, myelination delay, and cystic changes of the white matter and gliosis of the deep gray matter, which occurred months after acute neonatal hyperammonemia as a result of OTC deficiency [4]. Bilateral anterior temporal atrophy and high signal intensity at the temporal cortex have been observed in patients with late onset OTC deficiency, especially in the cingulate gyri and insular cortex [5]. Other MRI findings in OTC deficiency in patients with severe clinical manifestations such as somnolence, seizures, and hemiplegia included extensive infarct-like abnormalities, which were not present in our patient [6].

Besides routine MRI (T1 and T2 imaging), other neuroimaging modalities have been used to detect earlier measures of brain injury. Routine imaging may only detect damage at the macroscopic level when the patient is symptomatic and may lag behind clinical signs. Diffusion tensor imaging (DTI), which examines the disruption of white matter integrity, functional MRI (fMRI) which maps changes in brain hemodynamics that correspond 
to cognitive tasks, and proton magnetic resonance spectroscopy $\left({ }^{1} \mathrm{H}\right.$ MRS) which is able to detect the concentration of neurotoxic byproducts in various areas of the brain [7] have been used to characterize brain injury in patients with UCDs. In ${ }^{1} \mathrm{H}$ MRS, there is an increase in the glutamine/glutamate signal intensity accompanied by myo-inositol depletion. Other details of these advanced neuroimaging findings are beyond the scope of this case report.

Ammonia is converted to glutamine in astrocytes and being osmotically active, causes cytotoxic edema. It has been postulated by Kurihara et al. [5] that the T2 abnormalities on MRI may reflect diminished cerebral perfusion and early ischemia.

The neuroimaging findings in other causes of hyperammonemia differ from that due to UCDs. For example, in hepatic encephalopathy, classic MRI abnormalities include high signal intensity in the globus pallidum on T1-weighted images. The pattern of MRI abnormalities may thus also be able to guide the clinician in the workup for hyperammonemia [8].

With the knowledge of the specific neuroimaging findings of our patient, we are able to better determine the etiology and prognosis of focal epilepsy. She will probably need long-term anti-epileptic medications for seizure control.

\section{Conclusions}

To the best of our knowledge, the finding of bilateral mesial temporal sclerosis adds further evidence of the susceptibility of temporal lobes to injury from hyperammonemia. UCD can lead to mesial temporal sclerosis which leads to increased susceptibility of patients to seizures regardless of their metabolic state. This case also highlights the importance of neuroimaging in the workup of seizures in patients with UCD without metabolic decompensation.

Availability of data and materials

The dataset supporting the conclusions of this article is included within the article.

Authors' contributions

Acquisition of data - FW, DG, and HO. Analysis and interpretation of data - FW,

$D G$, and HO. Drafting of the manuscript - FW. Critical revision of manuscript - DG and HO. All authors read and approved the final manuscript.

Ethics approval and consent to participate

The authors declare that ethics approval was not required for this case report

Consent for publication

Written informed consent was obtained from the patient's parent for publication of this case report and any accompanying images. A copy of the written consent is available for review by the Editor-in-Chief of this journal.

\section{Competing interests}

The authors declare that they have no competing interests.

\section{Publisher's Note}

Springer Nature remains neutral with regard to jurisdictional claims in published maps and institutional affiliations.
Received: 28 September 2017 Accepted: 17 June 2018

Published online: 15 July 2018

\section{References}

1. Machado MC, Pinheiro da Silva F. Hyperammonemia due to urea cycle disorders: a potentially fatal condition in the intensive care setting. J Intens Care. 2014;2:22

2. Wolf NI, Bast T, Surtees R. Epilepsy in inborn errors of metabolism. Epileptic Disord. 2005;7(2):67-81.

3. Gropman AL. Patterns of brain injury in inborn errors of metabolism. Semin Pediatr Neurol. 2012:19(4):203-10.

4. Gropman AL. Brain imaging in urea cycle disorders. Mol Genet Metab. 2010; 100(Suppl 1):S20-30.

5. Kurihara A, Takanashi J, Tomita M, Kobayashi K, Ogawa A, Kanazawa M, et al. Magnetic resonance imaging in late-onset ornithine transcarbamylase deficiency. Brain and Development. 2003;25:40-4.

6. Mamourian AC, du Plessis A. Urea cycle defect: a case with MR and CT findings resembling infarct. Pediatr Radiol. 1991;21:594-5.

7. Gropman AL. Expanding the diagnostic and research toolbox for inborn errors of metabolism: the role of magnetic resonance spectroscopy. Mol Genet Metab. 2005;86:2-9.

8. Rovira A, Alonso J, Cordoba J. MR imaging findings in hepatic encephalopathy. AJNR Am J Neuroradiol. 2008;29(9):1612-21.

\section{Ready to submit your research? Choose BMC and benefit from:}

- fast, convenient online submission

- thorough peer review by experienced researchers in your field

- rapid publication on acceptance

- support for research data, including large and complex data types

- gold Open Access which fosters wider collaboration and increased citations

- maximum visibility for your research: over $100 \mathrm{M}$ website views per year

At BMC, research is always in progress.

Learn more biomedcentral.com/submissions 